\title{
Temporomandibular joint internal derangement: what we missed?
}

\begin{abstract}
Abbreviations: TMJ-ID; temporomandibular joint internal derangement, DCC; disco condylar complex, PRP; platelet rich plasma, HA; hyaluronic acid, LLLT; low level laser therapy
\end{abstract}

\section{Editorial}

Temporomandibular joint internal derangement (TMJ-ID) is the most common form of the TMJ disorders with broad non-surgical and surgical treatment modalities. The results of these different treatment modalities vary from study to study and from author to author resulting in confounding, frustrating, and unremitting dilemma. In view of the fact that, the disease is multi factorial, while our vision are constrained. TMJ-ID isn't just a disc displacement, coexisting disease problem includes; myofascial pain, mal-occlusion, clenching the teeth, the joint effusion, joint arthritis, change of Disc configuration, and LMP abnormality. Then, to achieve a better treatment result, the multifactorial concept should be in our mind with a broad vision to cover all aspects of the diseases process. Another important point, the success of the treatment of TMJ-ID doesn't depend only in treatment of the current joint problem. The treatment of the underlying possible causes will result in long-term success. Splint therapy is nonsurgical option for management of TMJ-ID with different outcome results. These different results give us a clue that we missed something regarding splint therapy. Before we use the splint for treatment of TMJ-ID, we have to ask our self, what we need from the splint?. Is it just to relive the symptoms? or to restore the normal disco-condylar complex (DCC)? Looking back in the literatures, thickness of the occlusal splint varies from $1 \mathrm{~mm}$ to $8 \mathrm{~mm}$. Increase the thickness of the occlusal splint lead to increase antero-inferior condylar movement which decrease overload on the retro-discal tissues and its elastic fibers, allowing adaptive changes to occur and giving the chance to healing in the elastic fiber which will pull the disk back to its normal position especially in the young age. Moreover, increase the thickness of the occlusal splint will increase the joint space and provides an occlusal relationship in the optimal masticatory system, and relax the attachment of the superior belly of the lateral pterygoid (LPM) to the disk. Looking back to the MRI of the TMJ, we should consider the relation of the LPM to DCC. In some cases, LPM inserted into the disc and pulled it forward, also the presence of the third head of the LPM has been reported. Furthermore, a close relationship has been reported between the functions of the LPM's and etiology of TMJ ID. Botulinum toxin-A injection to the LPM can block the muscle's impulse and temporarily weakens the contraction of muscle mostly improved the symptoms associated with the ID. And even-after, some patients showed less improvement, that's mean we missed something else? Since normal TMJ movements depend primarily on the disc freely sliding down the slope of the eminence, understanding how aberrations in the lubrication system contribute to TMJ dysfunction is clearly critically important. Subsequently, lubrication of the joint with Hyaluronic Acid (HA) or recently by injection of PlateletRich Plasma (PRP) which stimulates cell proliferation and the production of cartilage matrix by chondrocytes and bone marrowderived mesenchymal stromal cells and increases the production of
Volume 2 Issue I - 2015

\author{
Ayman Hegab \\ Faculty of Dental Medicine,Al-Azhar University, Egypt
}

Correspondence: Ayman Hegab, Clinical Associate Professor of Oral \& Maxillofacial Surgery, Faculty of Dental Medicine,AlAzhar University, Cairo, Egypt, Tel 0097433310124, Email hegab@.com prof.aymanhegab@yahoo.com

Received: November 21, 2014 | Published: November 27, 2014

hyaluronic acid by synoviocytes should be considered. Synergistic actions of using HA and PRP might result in immediate improvement contribute to the action of HA while long term results will depend on the action of the PRP. As we are looking for the best results, the longterm combined, structure modifying and symptom-modifying effects of glucosamine sulphate suggest that it could be of value in treatment of TMJ ID. We shouldn't miss the role of the concurrent cervical muscle spam and myofascial pain which can overstate the TMJ ID. Physiotherapy and Super pulsed low-level laser therapy (LLLT) seems to be a good choice as a non-invasive treatment for TMJ pain while exhibiting a low cost for the patient. Sir William Osler, noted physician (1849-1919), is reported to have said, "Listen to the patient; he is giving you the diagnosis." Psychological factors in terms of pain and interference with the quality of life should be considered. The view that occlusion and condyle position are the primary causes of TMD, and that diagnoses and treatments should be based on these philosophy, has been discredited. There is little to no evidence that treating subjects with TMJ-ID will prevent or mitigate future TMD. Bear in mind that, the treatment goals is to influence the repositioning of DCC to its normal position, most of the treating specialists focus on one of the treatment modalities. While, Combination of two or more non- invasive treatment modalities can offer better results. Finally, if the above mention treatment modalities failed, and before we choosing invasive surgical treatment modalities, please check again what we missed? I wish to thank the editors of Journal of dental health, oral disorders and therapy for inviting me to present this editorial.

\section{Funding}

None.

\section{Acknowledgments}

None.

\section{Conflicts of interest}

The authors declare that there was no conflict of interest. 